\title{
Sarcoma Care in an Urban Health-care System: Which Factors Lead to Variance of Care?
}

\author{
Alan T. Blank • Richelle C. Takemoto • Neeraj M. Patel • \\ Assad Baig • Timothy B. Rapp \\ Received: 30 August 2013 / Accepted: 11 December 2013 / Published online: 25 February 2014 \\ (C) Cobb/NMA Health Institute 2014
}

\begin{abstract}
Bone and soft tissue sarcomas are rare diagnoses in the orthopedic patient population. Without rapid evaluation and proper treatment, mortality is increased in these patients. The purpose of our study is to identify factors associated with a variance of care in bone and soft tissue sarcoma patients within a large urban hospital system. Our study retrospectively reviewed the charts of 117 patients with orthopedic tumors at both private and public hospital settings, and we recorded their treatment course. Race, insurance type, and hospital type were associated with variations in sarcoma care in our large urban hospital system. Surgeons should be aware of these variances when treating these groups of patients.
\end{abstract}

Keywords Orthopaedic oncology · Variance of care · Cancer

\section{Introduction}

Bone and soft tissue sarcomas are rare diagnoses in the orthopedic patient population. Documented rates of osteosarcoma have been recorded at three new cases per million per year [1]. With the use of modern neoadjuvant chemotherapy combined with surgery, the 10-year disease-free survival rate is approximately $60 \%$ in patients with localized disease versus $30 \%$ in patients with metastatic disease at diagnosis [2-4]. Without rapid evaluation and proper treatment, mortality risk is significantly increased secondary to a chance of metastasis [5].

\footnotetext{
A. T. Blank $(\bowtie)$

Department of Orthopaedics, NYU Hospital for Joint Diseases, 301

E 17th Street, 14th floor, New York, NY 10003, USA

e-mail: alan.blank@nyumc.org

R. C. Takemoto

Department of Orthopaedic Surgery, Allegheny General Hospital, Pittsburgh, PA 15212, USA
}

N. M. Patel $•$ A. Baig $\cdot$ T. B. Rapp

NYU Hospital for Joint Diseases, New York, NY, USA
Clinical studies have shown that not all tumors respond equally to the same treatment, and individual treatment plans are necessary for each malignancy [6].

Variance of care based on socioeconomic and racial factors has been well documented in the medical literature. Studies in medical oncology have documented a delay in diagnosis of cancer of up to 13 weeks on the average inpatient without insurance compared with privately insured patients [7]. The gynecology and surgical oncology literature have documented differences in health-care delivery and morbidity based on race and insurance type as well [8-10]. In the orthopedic literature, African American and Hispanic patients have been shown to have worse preoperative function before joint arthroplasty compared to Caucasian patients. This difference is believed to be due to delay in operative treatment in these minority groups $[11,12]$. Escalante et al. studied rates of total hip replacement and found that Hispanic patients with Medicare underwent joint replacement at much lower rates than non-Hispanic patients $[13,14]$. Although the medical literature has recently taken a focus on these social disparities, no studies have looked specifically into a variance of care in the orthopedic oncology patient population.

The identification of social factors associated with variations in care is a crucial step in creating optimal care for all orthopedic oncology patients. The purpose of our study is to identify demographic and clinical factors associated with a variance of care in bone and soft tissue sarcoma patients.

\section{Materials and Methods}

After institutional review board approval, we retrospectively reviewed 117 consecutive oncology patients with orthopedic tumors who presented between May 2008 and June 2011. Our orthopedic oncology department consists of a rotating resident team and one attending surgeon. Inclusion criteria for this study included diagnosis of a musculoskeletal sarcoma that 
had not yet been treated prior to presentation to our institution. Patients were excluded if they were lost to follow-up, received combined care in multiple facilities, or were found to have incomplete workup or treatment.

Of the 117 patients, $9 \%$ were lost to follow-up, $45 \%$ were diagnosed with a pathology other than sarcoma, and $22 \%$ of patients either had insufficient medical records or mixed care between facilities. Twenty-eight patients were diagnosed and treated for bone or soft tissue sarcomas and met all inclusion criteria. Our health-care system consists of four facilities that serve a large, diverse, urban population: large public, large private academic, and orthopedic specialty hospitals as well as an integrated cancer center. This wide array of clinical settings provides a useful background on which to conduct the present study.

We retrospectively reviewed patient demographics of interest including sex, race, insurance type, and hospital of treatment. Other variables of interest included studies obtained for workup and type of surgical and medical treatment. We also calculated a number of process indicating measures, including time from symptom onset to first visit, time from first visit to biopsy, time from first visit to pathology report, and time from first visit to surgery. Standard descriptive statistics, including mean and standard deviation (SD), were used to report baseline and demographic data. Univariate regressions, followed by stepwise multivariate regressions, were used to evaluate any association between demographic factors and our variables. Statistical significance was defined as $p<0.05$. All statistical analyses were performed with SAS 9.0 (SAS Institute, Inc., Cary, NC).

\section{Results}

Of the 28 patients that met inclusion criteria (14 males and 14 females), $44 \%$ were Caucasian, $26 \%$ Asian, $15 \%$ Hispanic, and $15 \%$ African-American. The health-care coverage for this population consisted of $58 \%$ private insurance, $22 \%$ Medicaid, $12 \%$ Medicare, and $8 \%$ self-pay. The most common diagnosis was osteosarcoma ( $25 \%$ ), followed by synovial sarcoma (18\%) and chondrosarcoma (11\%). Patients excluded from the study either did not care a malignant diagnosis or were lost to follow-up or sought care elsewhere. No patients who were excluded from the study died during their care process under our care.

When accounting for the aforementioned demographic variables in a multivariate model, Hispanic ethnicity was predictive of a longer delay between symptom onset and initial orthopedic visit (mean 134 days, $p=0.0065$, standard error (SE) 335.4), time from initial visit until pathology report (mean 242 days, $p=0.0028$, SE 36), and time from first visit until surgery (mean 230 days, $p=0.0024$, SE 33.8) (Fig. 1, Table 1). Self-pay was associated with less time from

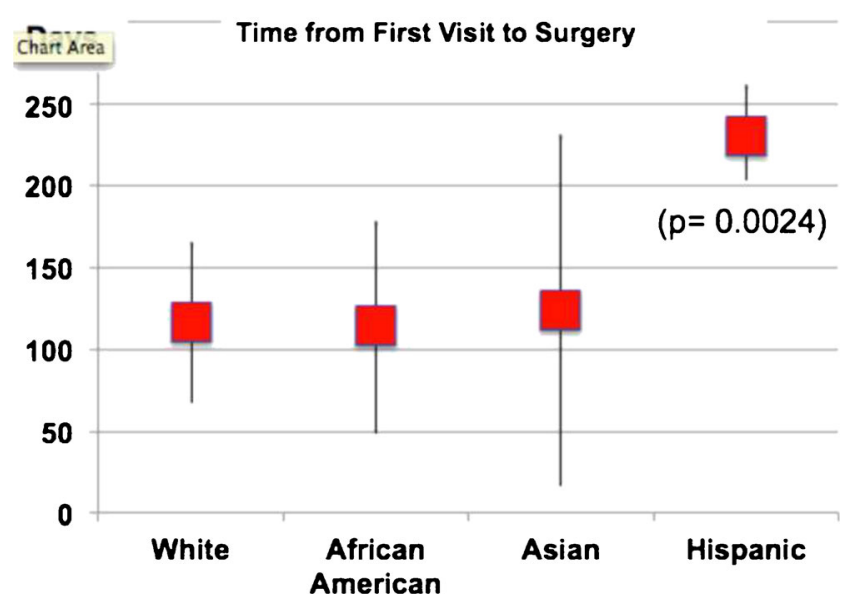

Fig. 1 Time from first orthopedic visit to time of surgery by patient race

symptom onset to first visit (mean 250 days, $p=0.038$, SE 583), but a longer time between first visit and pathology report (mean 260 days, $p<0.001$, SE 83.1) (Fig. 2). Female gender was predictive of a longer delay between first specialist visit and start of chemotherapy (mean 110 days, $p=0.039$, SE 14.8). Compared to those with private insurance, Medicare and Medicaid patients were more likely to undergo inpatient rather than outpatient workups $(60 \%, p=0.0063$, SE 0.19 , and $66 \%, p=0.038$, SE 0.18 , respectively). Compared to patients in our private facilities, public hospital patients waited longer from time of initial visit to diagnosis ( $p=0.012$, SE 43.1, mean 85 days longer) and from first visit to surgery $(p=0.017$, SE 60.1, mean 79 days longer) (Fig. 3). The time from symptom onset to first visit at our public hospital was 76 days longer, although no significance was found.

\section{Discussion}

Disparity in health care is well documented in the medical literature, and significant differences have been observed in multiple specialties [15-17]. Variance in care has been associated with race, insurance type, and gender in medicine, gynecology, general surgery, and orthopedic literature [9, 18-21]. These differences are important to recognize in order to improve health care for all patients. Our study found race, insurance type, and hospital type to be associated with variations in bone and soft tissue sarcoma care.

The most significant findings of our data were that Hispanic race was predictive of a longer delay between symptom onset and initial orthopedic visit, from initial visit until diagnosis of pathology, and from diagnosis to surgery. Though we have elucidated this association, the true etiology of these delays is unclear. It is possible that many providers have a level of unconscious bias that may contribute to some of the variances we have observed. We suspect that the documented differences are due to a combination of factors including 
Table 1 Statistically significant variables on bivariate analysis

\begin{tabular}{llll}
\hline Variables of significance & & & \\
\hline Dependent variable & Significant predictors & Mean (days or \%) & $p$ value (standard error) \\
\hline Symptom onset to first visit (days) & Hispanic race & 134 days & $0.0065(335.4)$ \\
& Self-pay insurance & 250 days & $0.0382(538.8)$ \\
First visit to pathology (days) & Self-pay insurance & 260 days & $<0.001(83.1)$ \\
First visit to chemotherapy (days) & Female sex & 110 days & $0.0391(14.8)$ \\
First visit to surgery & Hispanic race & 230 days & $0.0024(33.8)$ \\
& Public hospital & 178 days & $0.0017(60.1)$ \\
First visit to pathology (days) & Hispanic race & 242 days & $0.0028(36)$ \\
Inpatient vs outpatient workup & Medicare insurance & $60 \%$ & $0.0063(0.19)$ \\
& Medicaid insurance & $66 \%$ & $0.0381(0.18)$
\end{tabular}

inadequate communication in the patient's native language, access to the health-care system, temporary loss to follow-up, and differences of cultural expectations. Prior research has also found disparity of care among the Hispanic population. Some studies propose explanations for the differences observed, including folk and popular traditions of health care, communication and language barriers, educational differences, and roles of family members [22]. Physician-derived barriers to communication and patient respect have also been presumed to affect patient care. Some studies have documented the importance of cultural competence courses during medical training that may improve patient communication and understanding [23]. The Office of Minority Health, a division of the US Department of Health and Human Resources has established a set of guidelines for culturally and linguistically appropriate services in health and health care in order to help diminish these barriers we have demonstrated. These guidelines may be helpful in diminishing differences in care.

Our study also found that self-pay was associated with shorter time from symptom onset to first visit with an

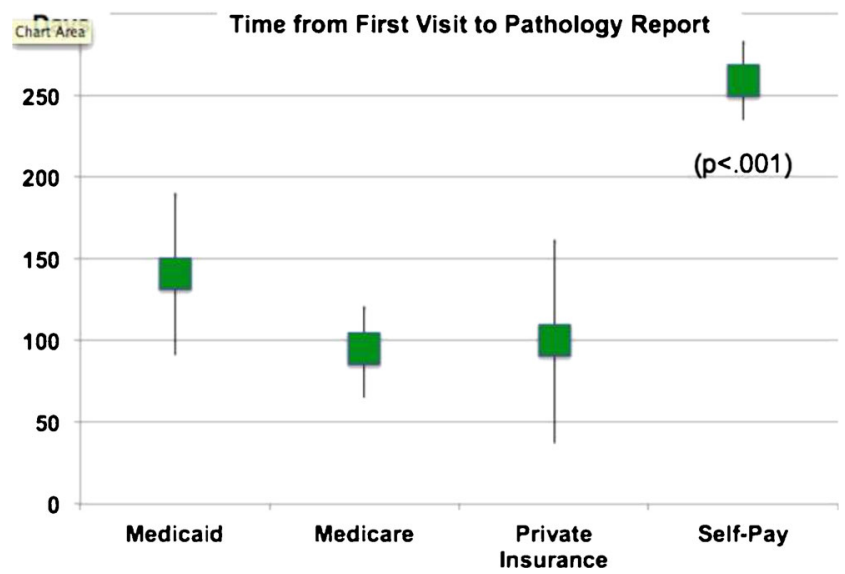

Fig. 2 Time from first orthopedic visit to time of pathology report by insurance type orthopedic specialist. However, this same group was also associated with longer wait from first visit with a specialist to time of diagnosis. Our group theorizes that this is due to the fact that those self-pay patients are able to present to emergency rooms with ease and will be able to see an orthopedist at that time. However, scheduling follow-up appointments in clinic and for procedures may prove more difficult for them. Medicare and Medicaid patients in our population were more likely to undergo inpatient rather than outpatient workups. We believe this is because Medicare and Medicaid patients often have difficulty obtaining outpatient specialist follow-up. It is plausible that the treating physicians decided to pursue inpatient workups in order to avoid losing these patients to follow-up. Ward et al. looked at cancer care utilization and insurance type and found that Medicaid and uninsured patients seen in emergency rooms had significant difficulty arranging follow-up appointments secondary to payment issues, and their time to diagnosis can therefore be delayed [24]. Halpern et al. performed a review of over 160,000 patients diagnosed with cancer and found that

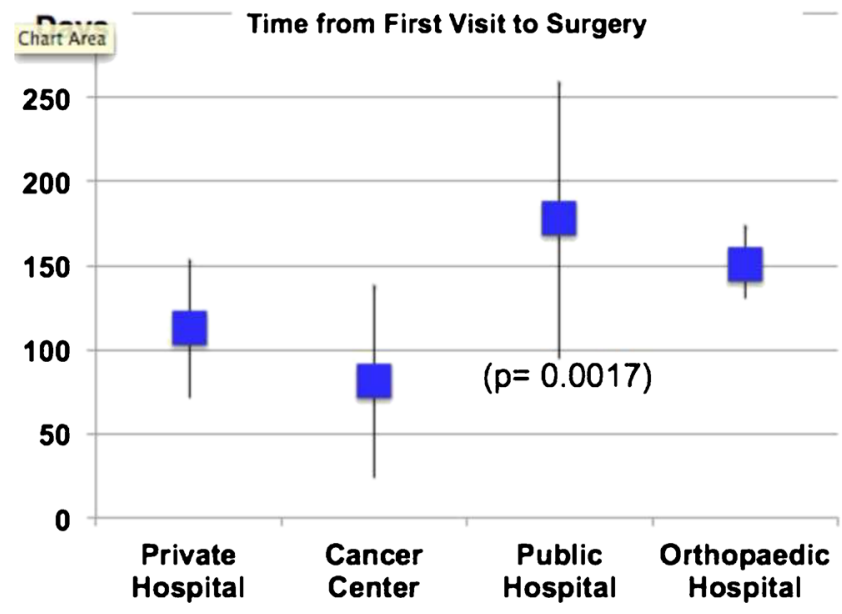

Fig. 3 Time from first orthopedic visit to time of surgery by hospital type 
diagnostic workups often took significantly longer when done as outpatient as opposed to inpatient [25]. We did not find a statistical correlation between location of workup and timing of treatment.

Our study found a significant difference in the time required for diagnostic workup and treatment in public versus private hospital settings. We also saw a notable trend in the time between symptom onset and first visit with a specialist. Yorio et al. found time from initial imaging to treatment to be nearly doubled in lung cancer patients treated at public hospitals compared to private hospitals [26]. Bedell et al. saw similar delays in breast cancer patients in public versus private hospitals [27]. Possible reasons for this disparity in care may be availability of resources, coordination of care, and payer administrative requirements.

The present study is not without limitations. Largely due to the relative rarity of musculoskeletal sarcomas, data was only available for 28 patients. A significant portion of our patients was lost to follow-up. If we were able to find more data from these patients, our results may have been altered. Furthermore, these patients did not have uniform diagnoses. Our orthopedic oncology team consisted of a single attending with a rotating group of residents. A number of other doctors were involved in the care from other specialties; therefore, clustering may have played a role in our data. With a limited sample size as well as time of study, we do not have any data relating to disease-free survival, progression-free survival, or any other prognostic factors. Our continued study of these patients may hopefully show more findings in the future. The retrospective nature of our data collection carries with it the inherent biases of this type of study design.

\section{Conclusion}

Despite these shortcomings, our study highlights factors leading to variance of care in orthopedic oncology patients in a diverse, urban hospital setting. Our findings are consistent with the other current literature regarding health-care disparities, but this is the first article to investigate these differences in orthopedic oncology patients. Our study found statistically significant delays in the care received by Hispanic patients as well as differences depending on insurance and hospital types. Surgeons should be aware of these variances when treating these patients. Future research should focus on elucidating the cause of these disparities, as they may have far-reaching implications at all levels of health-care infrastructure.

Conflict of Interest There are no conflicts of interest for any authors.

\section{References}

1. Campanacci M. Bone tumors. 2nd ed. Bologna: Lippincott; 1999. p. 1418-68.

2. Bacci G, Ferrari S, Bertoni F, et al. Long-term outcome for patients with nonmetastatic osteosarcoma of the extremity treated at the Istituto Ortopedico Rizzoli according to the IOR/OS2 protocol: an update report. J Clin Oncol. 2000;18:4016-27.

3. Huvos A. Bone tumors: diagnosis, treatment and prognosis. 2nd ed. Philadelphia: WB Saunders; 1991.

4. Bielack S, Kempf-Bielack B, Schwenzer D, et al. Neoadjuvant therapy for localized osteosarcoma of extremities. Results from the Cooperative Osteosarcoma Study Group COSS of 925 patients. Klin Padiatr. 1999;211:260-70.

5. Harris MB, Gieser P, Goorin AM, et al. Treatment of metastatic osteosarcoma at diagnosis: a Pediatric Oncology Group Study. J Clin Oncol. 1998;16:3641-8.

6. Longhi A, Errani C, De Paolis M, et al. Primary bone osteosarcoma in the pediatric age: state of the art. Cancer Treat Rev. 2006;32:423-36.

7. Martin S, Ulrich C, Munsell M, et al. Delays in cancer diagnosis in underinsured young adults and older adolescents. The Oncologist Epidemiology and Population Studies. 2007;12:816-24.

8. Sherman M, Devesa S. Analysis of racial differences in incidence, survival, and mortality for malignant tumors of the uterine corpus. Cancer. 2003;98(1):176-85.

9. Smotkin D, Nevadunsky N, Harris K. Histopathologic differences account for racial disparity in uterine cancer survival. Gynecologic Oncology. 2012;127:616-9.

10. National Cancer Institute. Cancer health disparities. http://www. cancer.gov/cancertopics/factsheet/disparities/cancer-healthdisparities

11. Slover J, Walsh M, Zuckerman J. Sex and race characteristics in patients undergoing hip and knee arthroplasty in an urban setting. $\mathrm{J}$ Arthroplasty. 2010;25:575-80.

12. Lavernia CJ et al. Race, ethnicity, insurance coverage, and preoperative status of hip and knee surgical patients. J Arthroplasty. 2004;19:978.

13. Escalante A et al. Disparity in total hip replacement affecting Hispanic Medicare beneficiaries. Med Care. 2002;40:451.

14. Escalante A et al. Recipients of hip replacement for arthritis are less likely to be Hispanic, independent of access to health care and socioeconomic status. Arthritis Rheum. 2000;43:390-9.

15. Schoenfeld A et al. The effect of race on outcomes of surgical or nonsurgical treatment of patients in the spine patient outcomes research trial (sport). Spine. 2012;17:1505-15.

16. Daumit GL, Hermann JA, Coresh J, et al. Racial disparity in cardiac decision making. Use of cardiovascular procedures among black persons and white persons: a 7-year nationwide study in patients with renal disease. Ann Intern Med. 1999;130:173-82.

17. Taylor BA, Casas-Ganem J, Vaccaro AR, et al. Differences in the workup and treatment of conditions associated with low back pain by patient gender and ethnic background. Spine. 2005;30:359-64.

18. Epstein A et al. Racial and ethnic differences in the use of high-volume hospitals and surgeons. Arch Surg. 2010;145(2): $179-86$.

19. Jordan JM, Lawrence R, Kington R, et al. Ethnic health disparities in arthritis and musculoskeletal diseases: report of a scientific conference. Arthritis Rheum. 2002;46:2280.

20. Novicoff W, Saleh K. Examining sex and gender disparities in total joint arthroplasty. Clin Orthop Relat Res. 2011;469:1824. 
21. Amaranto DJ, Abbas F, Krantz S, Pearce WH, Wang E, Kibbe MR. An evaluation of gender and racial disparity in the decision to treat surgically arterial disease. J Vasc Surg. 2009;50:1340-7.

22. Poon A, Gray K, et al. Cultural competence: serving Latino patients. J Pediatr Orthop. 2003;23:546-9.

23. Jimenez RL. Current activities in orthopaedic culturally competent care education. J Am Acad Orthop Surg. 2007;15:S76-9.

24. Ward E, Halpern M, Schrag N, et al. Association of insurance with cancer care utilization and outcomes. CA Cancer J Clin. 2008;58:9-31.
25. Halpern MT, Holden DJ. Disparities in timeliness of care for U.S. Medicare patients diagnosed with cancer. Curr Oncol. 2012 Dec;19(6):404-13.

26. Yorio JT, Xie Y, Gerber DE. Lung cancer diagnostic and treatment intervals in the United States: a health care disparity? J Thorac Oncol. 2009 Nov;4(11):1322-30.

27. Bedell MB, Wood ME, Lezotte DC, Sedlacek SM, Orleans MM. Delay in diagnosis and treatment of breast cancer: implications for education. J Cancer Educ. 1995;10:223-8. 